\title{
Palm Print Identification Using Improved Histogram of Oriented Lines
}

\author{
M. Arunkumar, S. Valarmathy \\ Department of ECE, Bannari Amman Institute of Technology, Sathyamangalam, Tamil Nadu, India \\ Email: arunkumarm@bitsathy.ac.in, atrmathy@rediffmail.com
}

Received 21 March 2016; accepted 15 April 2016; published 16 June 2016

Copyright (C) 2016 by authors and Scientific Research Publishing Inc.

This work is licensed under the Creative Commons Attribution International License (CC BY). http://creativecommons.org/licenses/by/4.0/

c) (†) Open Access

\begin{abstract}
Automatic palmprint identification has received much attention in security applications and law enforcement. The performance of a palmprint identification system is improved by means of feature extraction and classification. Feature extraction methods such as Subspace learning are highly sensitive to the rotation variances, translation and illumination in image identification. Thus, Histogram of Oriented Lines (HOL) has not obtained promising performance for palmprint recognition so far. In this paper, we propose a new descriptor of palmprint named Improved Histogram of Oriented Lines (IHOL), which is an alternative of HOL. Improved HOL is not very sensitive to changes of translation and illumination, and has the robustness against small transformations whereas the small translation and rotations make no change in histogram value adjustment of the proposed work. The experiment results show that based on IHOL, with Principal Component Analysis (PCA) subspace learning can achieve high recognition rates. The proposed method (IHOL-Cosine distance) improves $1.30 \%$ on PolyU I database, and similarly (IHOL-Euclidean distance) improves $2.36 \%$ on COEP database compared with existing HOL method.
\end{abstract}

\section{Keywords}

Histogram of Oriented Gradients, Histogram of Oriented Lines, Improved Histogram of Oriented Lines, Principal Component Analysis

\section{Introduction}

As a complementary technology for personal authentication methods, biometric identification is emerging as a powerful means for automatically recognizing identities. It concerns with identifying people by their physiological individuality such as its retina, iris pattern, fingerprint, palmprint, hand geometry and face on some behavioral aspects of voice, signature and gesture. For past decades, face, voice and palmprint identification have been widely used. A numerous biometrics have been successfully developed for different applications like 
ATM, colleges and school, public sectors, surveillance, airport security control and law enforcement [1].

Palmprint methods have advantages like convenient, reliable, efficient and secure. It has fast recognition rate and speed. In recent times, palmprint is espoused for recognition by the researchers [2]. Palmprint is composed of three main kinds of features namely principal lines (generally three palm lines on the palmprint), wrinkles (weaker and irregular lines) and finally minutia (valley and ridge feature which is alike to those in finger prints). The texture and lines are the lucid feature at low resolution $(<100 \mathrm{dpi})$. A numerous techniques are introduced for palmprint recognition. The coding based approaches like line feature [3], feature points [4], Fourier spectrum [5], Eigen palm feature [6], texture energy [7], wavelet signature [8], gabor phase [9], competitive code [10], statistical line based [11], palm code etc. However, these need high standardization palmprint representation. It has been solved by using ordinal measure for common print representation [12].

Subspace learning methods such as Principal Component Analysis (PCA) [13] and Linear Discriminant Analysis (LDA) are extensively used for last two decades. These methods are not very much sensitive to rotation, illumination, translation and variance even if small variance values are appeared. Fusion based wavelet representation [14] mainly used to extract the dominant principal wrinkles and principal lines from over complete wavelet extension sub bands. These palmprint features are recognizing more effectively with low computational cost than other traits.

\section{Literature Review}

Local Binary Pattern (LBP) [15]-[17] encodes the difference between the center pixel and neighbor ones in circular sequence manner. It is very much sensitive to noise such as brightness variation in real picture. It computes large feature length for classification. Local Ternary Pattern (LTP) [18] can calculate the feature length of LBP by means of extended 3 values. LTP is less sensitive to noise.

Local Derivative Pattern (LDP) [19] proposed for obtaining the change of derivative direction among local neighbors and encodes the turning point in given direction. LDP is suffers more to higher order noise than LBP. Local Gabor Binary Pattern Histogram sequence (LGBPHS) [20] which merges the local binary pattern and magnitude of Gabor filter. It is robust to local image transformation caused by variation of illumination and noise.

To overcome these problems, Ma et al. [21] combined the Gabor filter with LBP. It is effectual but takes large computational time and cost. Ekinci and Aykut [22] proposed a novel palmprint recognition technique by integrating kernel Prinicipal Component Analysis and Gabor Wavelet Representation. The main disadvantage of GWR is high computational cost. Chu and Lie [23] take out the palm line and orientation features. They used the directional wavelet depiction. Later, Zhang and Tao [24] extract the orientation and line features effectively. They espoused radon transform and take out the Directional Representation (DR) of palmprint. This method holds a stronger discriminative information, and is sensitive to illumination change. Still, their sensitivity, translation, rotation and variance are not been solved. From the exceeding investigation, the palmprint recognition methods are mostly due to translation, rotation and illumination. HOG [25] is applied using locally normalized histogram of gradient orientations features alike to SIFT descriptors. It provides good outcome for person detection.HOG drawbacks are as follows: Gradient in HOG is not fine mean to detect the orientation of pixels and line responses. Because of different palm line have different width and there are more complicated intersections between lines.

Wei Jia [26] proposed HOL algorithm for palmprint recognition with Gabor and radon filter. It has advantages of invariance to change in illumination. It is robust to translation and rotation which makes only small change in histogram value. It performs well compare to Directional Representation, Gabor Wavelet Representation, Original representation and Local Binary Pattern. But still it has annoying Direct Component.

In this paper, we proposed an improved HOL algorithm using improved gabor filter. The natural images are better coded when viewed on logarithmic frequency scale. The improved HOL works on logarithmic frequency scale. It eliminates the annoying DC component. It improves its recognition accuracy by dividing palm image into sub region.

\section{Proposed Feature Extraction Algorithms Improved Gabor Filter}

Figure 1 shows the block diagram of palmprint identification system. In the paper [26], Gabor filter is used to identify the orientation and line response of pixels. An alternative to the Gabor filter is the improved Gabor filter. 


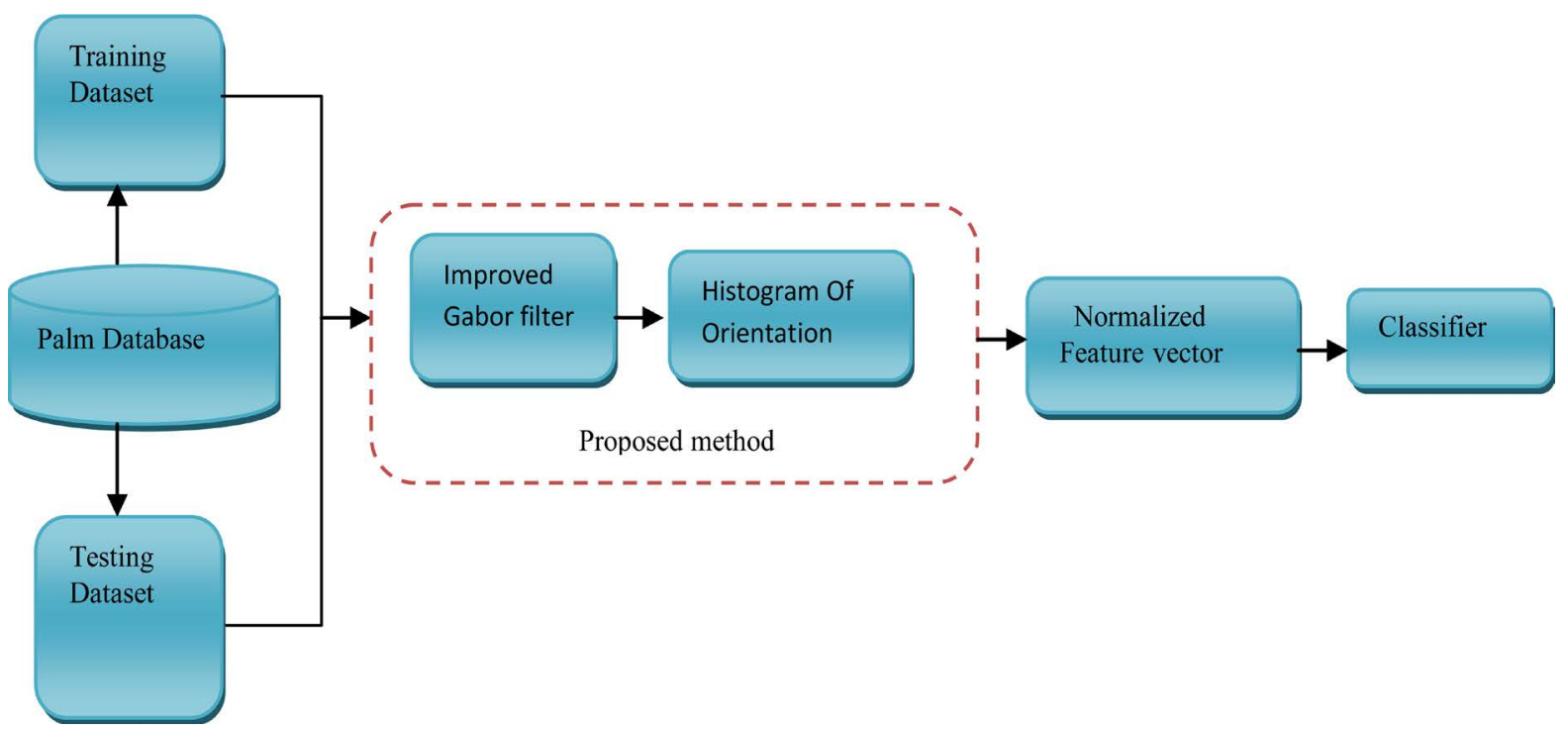

Figure 1. Block diagram of palmprint identification system.

The usual images can be well coded by this improved Gabor filter. It has Gaussian transfer function when viewed on the log frequency scale. However the Gabor filter has the transfer function when viewed on linear frequency scale. There are three main advantages of improved Gabor filter over Gabor filter. To begin with, images with wider range of spectrum cannot be filtered using single Gabor filter as the bandwidth is limited to one octave. However it is not in the case of improved Gabor. Whose bandwidth is extended that reduces the use of many filters. Secondly, it has no DC component and so the palm features can be extracted regardless of background brightness. Hence it reimburses the variations in illumination. Finally, Gabor filter center more at low frequency that causes the redundant information. On the other hand this filter overcomes it by having the extended tail which in excess of represents the high frequency components. Thus Improved Gabor filter is preferred over the Gabor filter in our improved HOL method. The Transfer function of improved Gabor filter consists of two components: angular component, which controls the orientation that the filter responds and similarly radial component, which controls the frequency band. These two gets multiplied to obtain the overall filter. The transfer function in the frequency domain is given as:

$$
I G(f, \theta)=I G(f) * I G(\theta)
$$

where $I G(f, \theta)$ in (2) represents the filters center frequency is $f$ and orientation angle of Improved Gabor filter is $\theta$; sigmaf is termed as radial bandwidth which is the ratio of standard deviation of Gaussian to the center frequency, thetasigma is termed as angular bandwidth to calculate the standard deviation of the angular Gaussian function used to construct the filter in frequency plane.

$$
I G(f, \theta)=\exp \left(\frac{(-\log (r / f))}{2 * \log (\text { sigmaf })}\right)^{2} * \exp \left(\frac{(- \text { dtheta })^{2}}{2 *\left((\text { thetasigma })^{2}\right)}\right)
$$

The Filter bank of Improved Gabor filter is created with four scales and six orientations to extract features from the palm images. It results 24 different filter transfer functions that denotes different scales and orientations as shown in Figure 2.

The image is converted in the frequency domain to make the process faster than in spatial domain. Since convolution takes more time in spatial domain. Then the 2D FFT applied image is multiplied with the transfer functions results the 24 spectral representations for each image. Finally the spectra are then transformed into the spatial domain using 2Dinverse FFT and this process produces feature arrays of larger number. For sigmaf as 0.75 bandwidth is limited to one octave whereas sigmaf $=0.55$ extends the bandwidth to two octaves. The value of angular bandwidth is set as thetasigma $=1.5$ to get even spectral coverage. Thus the orientation components of this improved gabor filter make the filter robust to different orientations. 


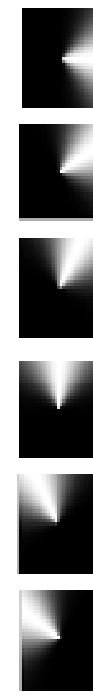

(a)

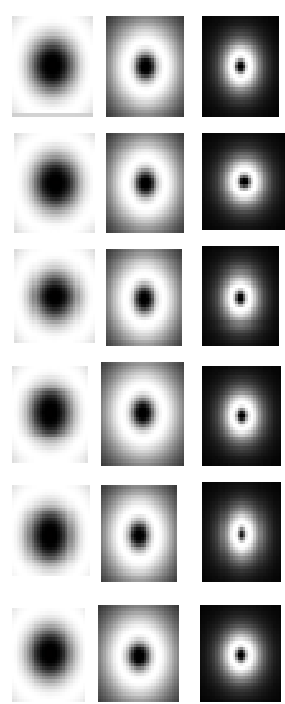

(b)
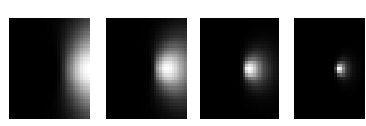

0

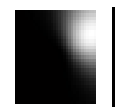

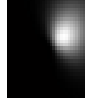

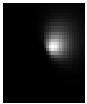

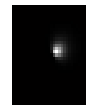

0

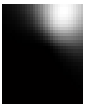

4
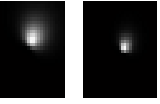

0

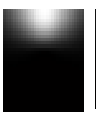

4
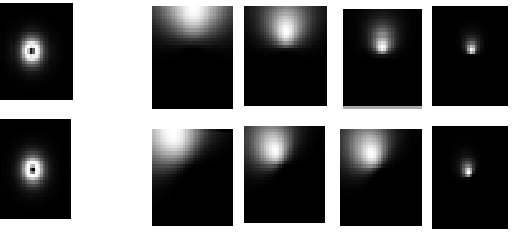

0
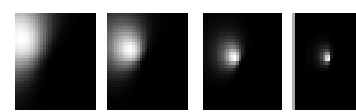

Figure 2. Improved gabor filter components with (a) 6 orientations and (b) 4 scales for each orientation (c) overall filter by multiplying both the angular and radial components.

\subsection{HOL Descriptor Using Gabor Filter}

HOL algorithm was proposed by Wei Jia et al. (2014) [26]. The Gabor Filter can be represented as a line shape filter to extract the orientation and line features of palmprint images.

2-D Gabor filter can be represented in general equation form:

$$
G(x, y, \theta, u, \sigma)=\frac{1}{2 \pi \sigma^{2}} \exp \left(-\frac{x^{2}+y^{2}}{2 \sigma^{2}}\right) \exp (2 \pi i(u x \cos \theta+u y \sin \theta))
$$

where $i=\sqrt{-1} ; \theta$ represents the orientation of the function; $u$ represents as the frequency of sinusoidal wave and $\sigma$ represents as the standard deviation of the Gaussian cure in (3). General algorithm for extracting orientation and the line response of the pixels in the palmprint image is as follows:

Step 1: Convolute the image I with the real part of gabor filter to generate the gabor filter bank.

Step 2: Find the orientation and magnitude response for each of the pixels in an image.

Step 3: Obtain the histogram and finally perform the normalization. Thereby descriptor can be generated.

\subsection{Proposed HOL Descriptor Using Improved Gabor Filter}

In [26], Gabor filter is used to extract the line response and orientation of pixels. In this work an Improved Gabor filter is used instead of Gabor filter to generate the HOL descriptor because of the advantages described in the previous section. Based on the transfer function, Improved Gabor filter bank is generated with four scales and six orientations. Let the palmprint image be I, the steps for extracting the line response and orientation of pixels using the filter bank is explained as follows:

Step 1: Generate the filtered images by convoluting the palmprint image I with the designed improved gabor filter bank which is represented in Figure 3.

Step 2: Then divide the obtained image into $\mathrm{m} \times \mathrm{m}$ non overlapping cells.

Step 3: By integrating b1 $\times$ b2 cells, a block is constructed first and overlap the adjacent blocks which is represented in Figure 4.

Step 4: The magnitude $m(x, y)$ and orientation $\theta(x, y)$ response for each pixel can be found using the equations given below:

$$
\begin{gathered}
m(x, y)_{I . \text { Gabor }}=\min \left(I(x, y) * I G\left(X, Y, \theta_{n}\right)\right) \\
\theta(x, y)_{I . \text { Gabor }}=\arg \min _{n}\left(I(x, y) * I G\left(x, y, \theta_{n}\right)\right)
\end{gathered}
$$




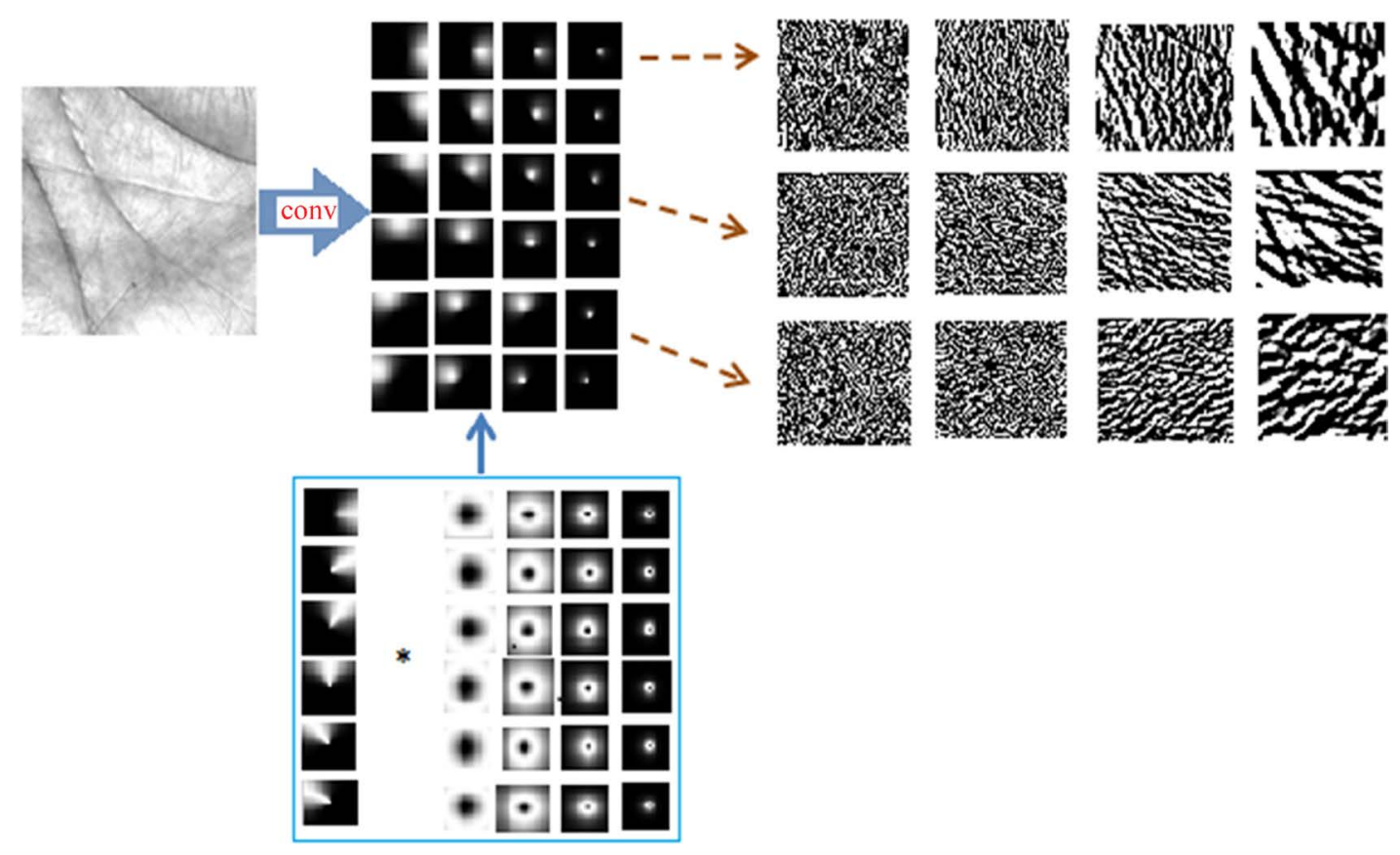

Figure 3. Generation of filtered images.

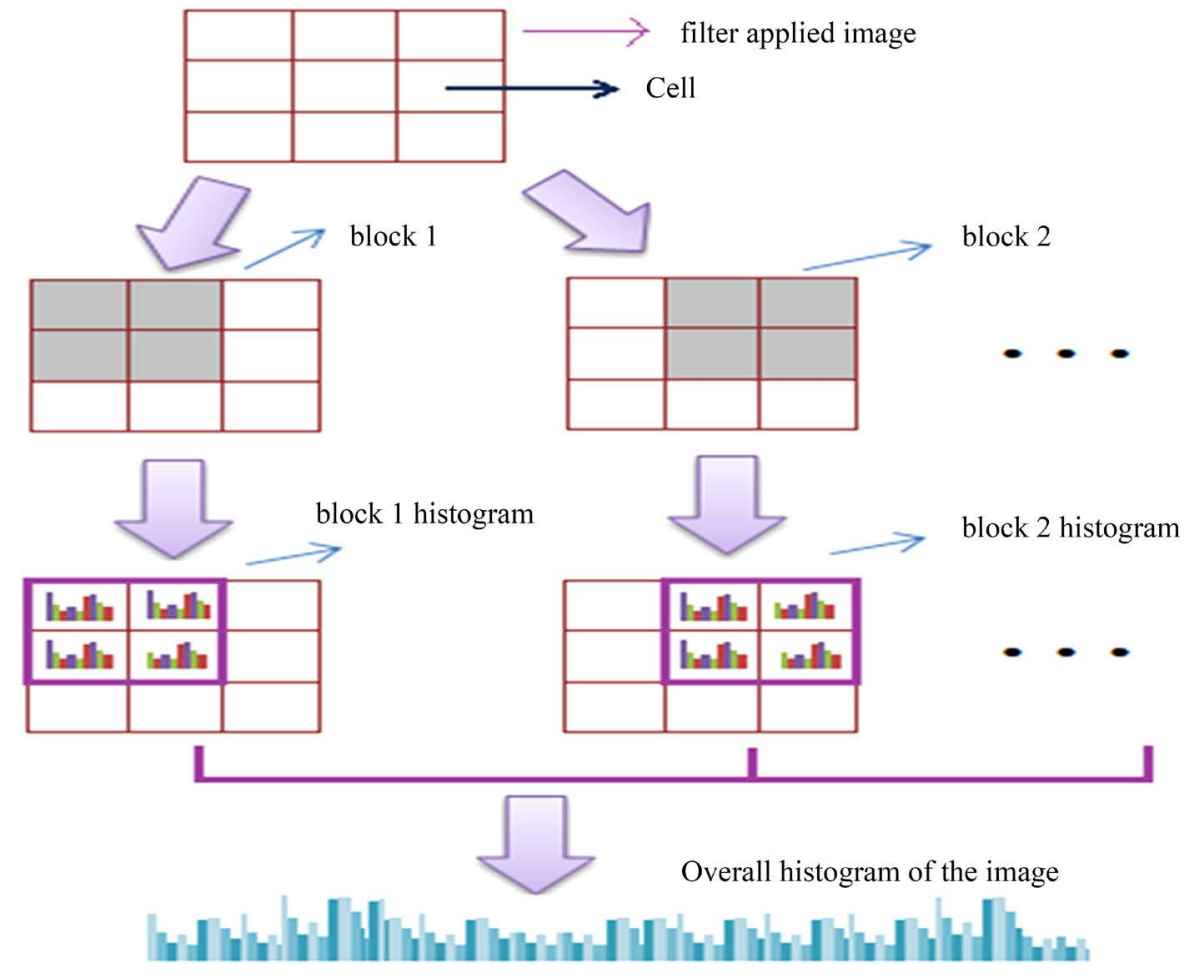

Figure 4. Generation of overall histogram.

where * denotes the convolution operator and $I G$ denotes improved gabor filter.

Step 5: Separate the orientation range of $\left(0^{\circ}\right.$ to $\left.180^{\circ}\right)$ into $\mathrm{n}$ bins and then calculate the histogram within cell.

$$
H C(n)_{i}=H C(n)_{i}+m(x, y)
$$

if $I(x, y) \varepsilon_{\text {ceell }}$ and $\theta(x, y) \in \operatorname{bin}(n)$. 
Step 6: By integrating all the HC's within a block $(2 \times 2$ cells as a block $)$, histogram for the block is obtained which is represented as:

$$
H B_{j}=\left\{H C_{1}, H C_{2}, \cdots, H C_{b 1^{*} b 2}\right\}
$$

Step 7: Then do L2-norm block normalization to normalize the vector $H B_{j}$

$$
N H B_{j}=\frac{H B_{j}}{\sqrt{\left\|H B_{j}\right\|_{2}^{2}+v}}
$$

In general, normalization of the feature vector is obtained as:

$$
\text { Feature vector }=\text { feature vector } /\left(\operatorname{sqrt}\left(\text { norm }(\text { feature vector })^{2}+v\right)\right)
$$

where $v$ is a constant which is too small used to eliminate the problem of division by zero. Then the histogram for the whole image (HOL descriptor) with $\mathrm{N}$ blocks is calculated by integrating all the normalized block histograms as shown in Figure 4 and is given by,

$$
H O L_{\text {descriptor }}\left\{N H B_{1}, N H B_{2}, \cdots, N H B_{j}, \cdots, N H B_{N}\right\} .
$$

\section{Experimental Results and Discussion}

\subsection{PoyU I Database}

Images in Figure 5 denote the six palmprint ROI image of PolyU I database, which were collected from the similar palm but at two dissimilar sessions. Three images in Figure 5(a) were captured in first session and the other three images in Figure 5(b) were captured in second session. It is to be observed that there are also variations in illumination between the images captured at two sessions. Before the experiments HOL using Gabor and improved Gabor are conducted, the parameters such as size of the cell and the number of bins $n$, the value of filter size and theta in gabor filter, the value of frequency and orientation should be predetermined.

For conducting the experiments, the size of the cell is selected as $16 \times 16$ and the number of bins $\mathrm{n}$ is espoused as 9. Consequently for the ROI palmprint image with the size of $128 \times 128$, there are entirely 64 cells i.e. $8 \times 8$ cells along with 49 blocks i.e. $7 \times 7$ blocks. For HOL using Gabor, the value of filter size and theta is determined as 2 and 45 respectively. The main parameters of proposed algorithm are the frequency and orientation which control the frequency and bandwidth of the filter respectively. These optimal values of selection make the algorithm to achieve the higher recognition rate. To reduce the size of the feature vector PCA has been applied. The recognition rates of existing and the proposed IHOL are obtained using PCA as a dimensionality reduction technique. For instance, feature vector size obtained from improved HOL algorithm on the PolyU I database is 1 $\times 8100$ features. By means of dimensionality reduction technique PCA, the feature vector size is reduced to different extent.

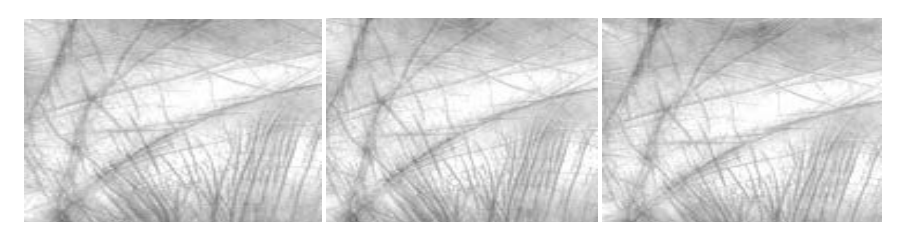

(a)

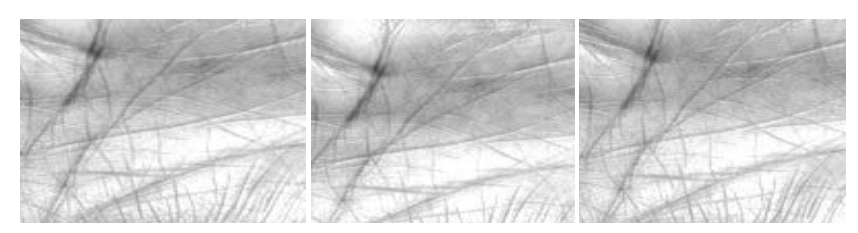

(b)

Figure 5. Six palmprint ROI images from the same palm in PolyU I database. (a) Three images captured in the first session; (b) In the second session next three image is captured. 
We have simulated existing algorithm such as HOG and HOL and proposed algorithm IHOL with different feature size using principle component analysis. Table 1 shows that using IHOL using vector-based subspace learning method attaining higher recognition rates. While feature dimension ranges from 100 to 200 , the recognition rate of proposed method is $97 \%$, which is a very optimistic recognition.

On comparing with HOG and HOL, higher recognition rate is obtained through IHOL algorithm. It is also to be noted that the maximum recognition rate for existing HOL algorithm is obtained when the dimension is 150 . Below the dimension of 100 , recognition rate is marginally decreasing which are represented in Table 1.

In the existing method, $u$ (frequency) is the parameter that controls the bandwidth of the filter. In the same mode, the value of frequency (f) and orientation $(\theta)$ of our improved gabor filter are to be changed and notice the far better values which give higher recognition rate. Table 2 shows that for the proposed algorithm, different wavelength (inverse to the frequency) values changing from 0.001 to 2 .

Table 3 shows the orientation (controlling bandwidth of filter) values ranging from pi/6 to $6 \mathrm{pi} / 6$ (6 orientations) are used to obtain optimal recognition value. The wavelength values greater than or less than 0.01 , the performance of the filter is decreasing which is depicted in Table 2. The filter outperforms well when the orientation value is $4 \mathrm{pi} / 6$ depicted in Table 3. Hence, the determined wavelength value as 0.01 and orientation value as $4 \mathrm{pi} / 6$.These values provide the higher recognition rate.

Table 4 shows that the identification performance of the proposed method. IHOL performs well when the training samples are increased from 500 to 700 in POLYU database. This result reveals that the proposed method is suitable for identification. We have simulated existing algorithm such as HOG and HOL and proposed algorithm IHOL with different training and testing samples.

Table 1. Recognition rate at different dimensions on PolyU I database.

\begin{tabular}{ccccc}
\hline \multirow{2}{*}{ S. No } & \multirow{2}{*}{ Dimension } & \multicolumn{3}{c}{ Recognition rate \% } \\
\cline { 3 - 5 } & & HOG & HOL & Improved -HOL \\
\hline $\mathbf{1}$ & 30 & 92.50 & 93.50 & 95.33 \\
$\mathbf{2}$ & 50 & 92.75 & 93.75 & 96.67 \\
$\mathbf{3}$ & 80 & 93.65 & 94.75 & 96.87 \\
$\mathbf{4}$ & 100 & 94.65 & 95.75 & 97 \\
$\mathbf{5}$ & 150 & 95 & 96 & 97 \\
$\mathbf{6}$ & 200 & 95 & 96 & 97 \\
\hline
\end{tabular}

Table 2. Optimum recognition rate at different.

\begin{tabular}{ccc}
\hline S. No & Wavelength & Recognition rate \% \\
\hline $\mathbf{1}$ & 2 & 88.667 \\
$\mathbf{2}$ & 1.5 & 89.667 \\
$\mathbf{3}$ & 1 & 90 \\
$\mathbf{4}$ & 0.1 & 96 \\
$\mathbf{5}$ & 0.01 & 97 \\
$\mathbf{6}$ & 0.001 & 96 \\
\hline
\end{tabular}

Table 3. Optimum recognition rate at different orientations.

\begin{tabular}{ccc}
\hline S. No & Orientation & Recognition rate \% \\
\hline $\mathbf{1}$ & 30 & 81.333 \\
$\mathbf{2}$ & 60 & 87.333 \\
$\mathbf{3}$ & 90 & 96.333 \\
$\mathbf{4}$ & 120 & 97.000 \\
$\mathbf{5}$ & 150 & 96.667 \\
$\mathbf{6}$ & 180 & 96.337 \\
\hline
\end{tabular}




\subsection{Experimental Results on the COEP Database}

Before the experiments are conducted in the COEP database, preprocessing is done to extract the region of interest (ROI) of the palm for feature extraction. The ROI is to be determined so that there is maximum variation among different users. To extract the ROI, the steps followed are: 1) Altering the RGB image to the gray scale image; 2) Using the position tracing algorithm, identify the specified ROI 3) Using the dimensions obtained with height and width, extract the ROI form the palm image.

The dimension of palm images in the database is $1600 \times 1200$. After perceive the ROI, the palmprint is cropped. The palmprint image is resized to $128 \times 128$ of resolution. The ROI is obtained from the palm image in COEP database is depicted in Figure 6.

Images in Figure 7 signify the eight palmprint ROI images of COEP database, which is gathered from the same palm at two dissimilar sittings. Four images in Figure 7(a) were captured during first session and the remaining four images in Figure 7(b) were captured during second session. It can be seen that there are also variations in illumination between the images captured at two sessions. The first four palmprints in the first row Figure 7(a) are used for the training phase and the next four palm prints in the second row Figure 7(b) are used for the testing phase. Therefore, the total number of images for the training and the testing sets are 652 and 652 respectively.

For the experiments such as HOL using gabor and proposed algorithm, the same parameters are adopted as mentioned in the experiment onPolyU I database. The frequency and orientation parameters is set as same as used in PolyU I database because the filter performance on COEP database is far closer to the performance on

Table 4. Varying the training and testing samples on PolyU I database.

\begin{tabular}{cccccc}
\hline \multirow{2}{*}{ S. No } & \multicolumn{5}{c}{ Recognition rate $\%$} \\
\cline { 2 - 6 } & Training Samples & Testing Sample & HOG & HOL & IHOL \\
\hline $\mathbf{1}$ & 500 & 500 & 90.200 & 92.40 & 94.20 \\
$\mathbf{3}$ & 600 & 400 & 91.700 & 93.75 & 95 \\
\hline
\end{tabular}

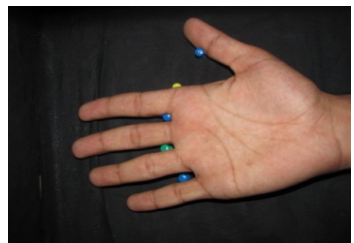

(a)

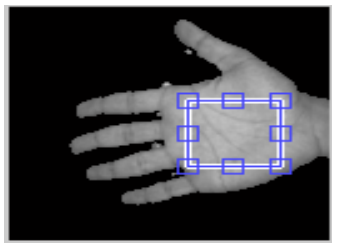

(b)

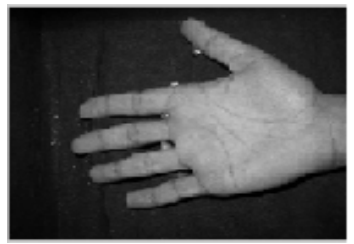

(c)

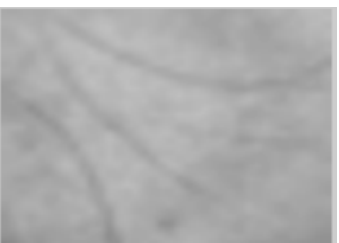

(d)

Figure 6. Extraction of ROI from palm image in COEP database: (a) Original palmprint image; (b) Color image converted into grayscale image; (c) ROI extraction; (d) Extracted palmprint.
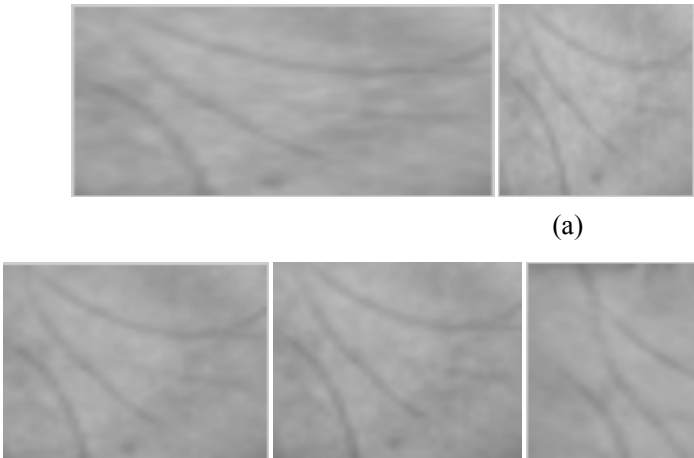

(a)
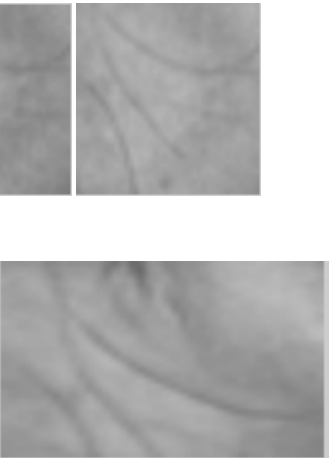

(b)

Figure 7. Eight palmprint ROI images from the same palm in COEP database: (a) Four image captured in first session; (b) Four image captured in second session. 
PolyU database. Here also, we undergo Principal Component Analysis (PCA) as a dimensionality reduction technique to condense the size of the feature vector.

The recognition rates of HOG, HOL and the proposed IHOL can be obtain while using Principal Component Analysis (PCA) as a dimensionality reduction technique on the COEP database. On comparing the three algorithms, higher recognition rate is obtained through IHOL algorithm. It is also to be noted that the maximum recognition rate for the existing HOL algorithm is obtained when the dimension is 150 . However, maximum recognition rate in our work is obtained when the dimension is between 100 and 200.

Below the dimension of 100 , recognition rate is marginally decreasing which are represented in Table 5 . Recognition rate of three different algorithms worked on COEP database is shown in Table 6 and it depicts that our improved HOL algorithm has a good recognition performance than the other two algorithms.

\subsection{Discussion}

PolyU I database is a challenging database because there are several changes of illumination between the images in different sessions. The subspace learning method using the proposed achieves better recognition rate compared to existing method. It could be decided that proposed method is evincing robust to slight illumination. However recognition of proposed work is very different for two databases.

In this paper, we have mentioned that the proposed method has the robustness against small transformation because of rotation and shift values make only small changes in histogram values. For example, in our work, the size of a cell is $16 \times 16$, so the size of a block which contains $2 \times 2$ cells is $32 \times 32$. In Figure 8(a), the block in the center area of palm image is represented as a black square.

In Figure 8(b) and Figure 8(c), block area after rotation of $-5^{\circ}$ and translation of three pixels respectively. The histogram values are varied in small after rotation and translation of palm print image. Therefore, it is simple to understand why our proposed work helps subspace learning method to reach desirable recognition performance. Here, some standard identification experiment for PCA techniques using PolyU I and COEP database to show that our improved method is robust to small transformations proposed method.

Table 5. Recognition rate at different dimensions on COEP database.

\begin{tabular}{ccccc}
\hline \multirow{2}{*}{ S. No } & Dimension & \multicolumn{3}{c}{ Recognition rate \% } \\
\cline { 2 - 4 } & $\mathbf{1}$ & HOG & HOL & Improved -HOL \\
$\mathbf{2}$ & 50 & 78.232 & 79.081 & 81.021 \\
$\mathbf{3}$ & 80 & 78.006 & 79.590 & 81.391 \\
$\mathbf{4}$ & 100 & 78.850 & 80.050 & 81.998 \\
$\mathbf{5}$ & 150 & 79.800 & 80.312 & 82.209 \\
$\mathbf{6}$ & 200 & 79.800 & 80.312 & 82.209 \\
\hline
\end{tabular}

Table 6. Varying the training and testing samples on COEP database.

\begin{tabular}{|c|c|c|c|c|c|}
\hline \multirow{2}{*}{ S. No } & \multirow{2}{*}{ Training Samples } & \multicolumn{4}{|c|}{ Recognition rate \% } \\
\hline & & Testing Sample & HOG & HOL & Improved -HOL \\
\hline 1 & 652 & 652 & 76.50 & 78.60 & 80.97 \\
\hline 2 & 815 & 489 & 77.89 & 80.31 & 82.209 \\
\hline 3 & 738 & 326 & 78.88 & 80.31 & 82.204 \\
\hline
\end{tabular}

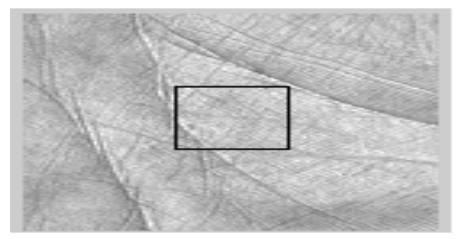

(a)

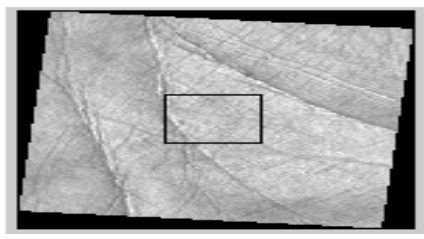

(b)

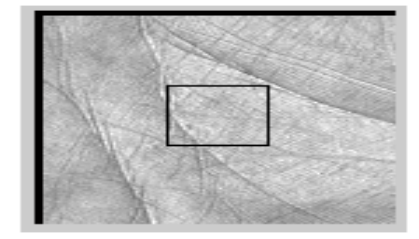

(c)

Figure 8. Different representations of image: (a) original image; (b) image after rotation; (c) image after translation. 
In the first experiment, the training set is 700 images and the testing set is 300 images in probe rotate $k(k=$ $\left.-1^{\circ},-2^{\circ},-3^{\circ},-4^{\circ},-5^{\circ}\right)$. For above mentioned methods of two databases and its recognition rate are shown in Figure 9. These result clearly display the small rotation have the limited effect for the existing method when compared to.

In the second experiment, the training set and train set is unchanged in probe to set shift $m(m=-1,2,3,4,5)$ pixels towards left in Figure 8(c) for HOG, HOL, and improved HOL method of PolyU and COEP database and its recognition rate are shown in Figure 10. These result clearly display the small shift have the limited effect for the existing method when compared to proposed method.

Figure 11 shows that IHOL works better with cosine distance compare to euclidean distance measure on PolyU I database. It depicts that our improved HOL algorithm has attain good recognition rate when compare to HOL algorithm in PolyU I database. In the palmprint classification stage, matching features using the Cosine distance is far better than the Euclidean distance on PolyU I database. Similarly that IHOL works better with euclidean distance compare to cosine distance measure on COEP database. It depicts that our improved HOL

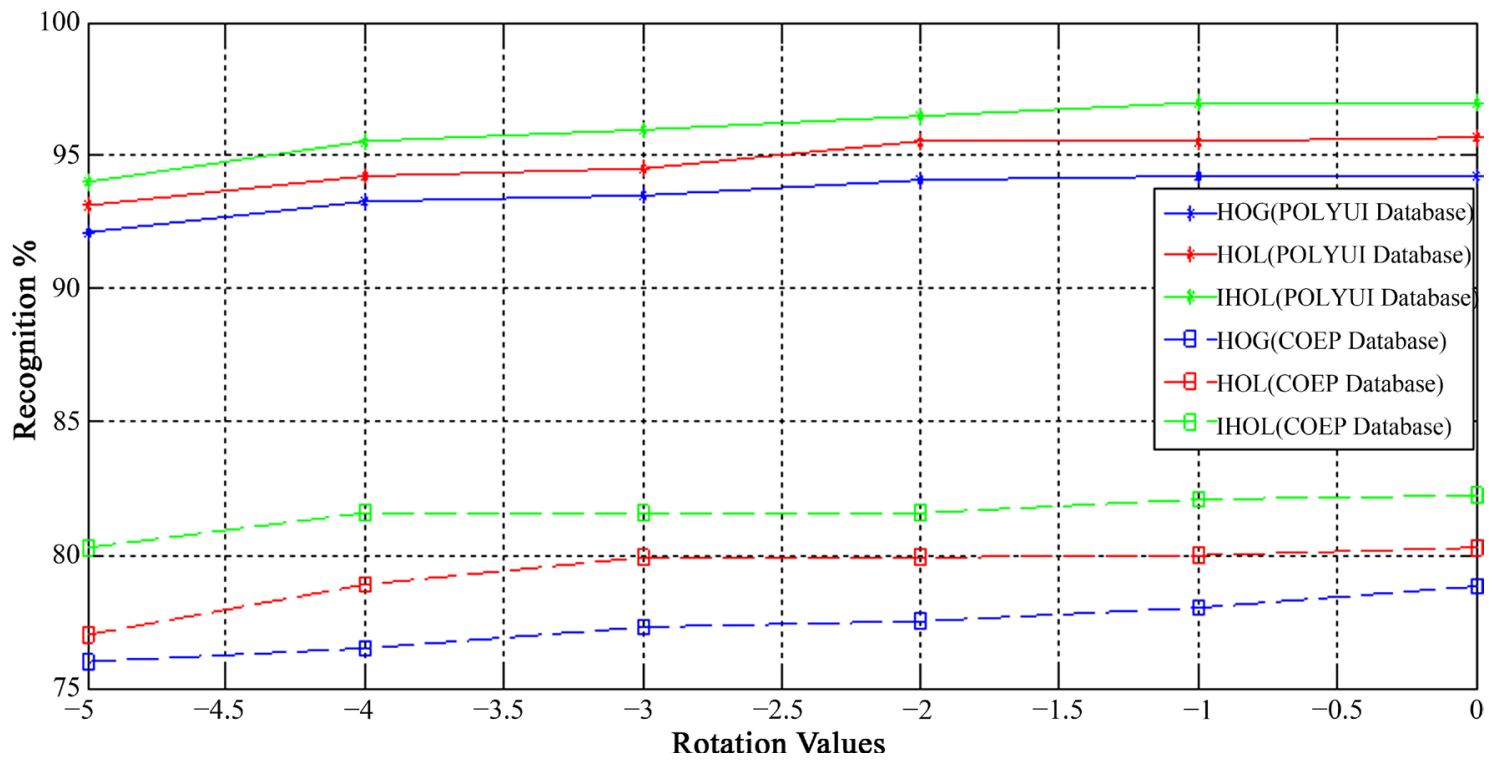

Figure 9. Recognition rate for different shift values on POLYU I/COEP database.

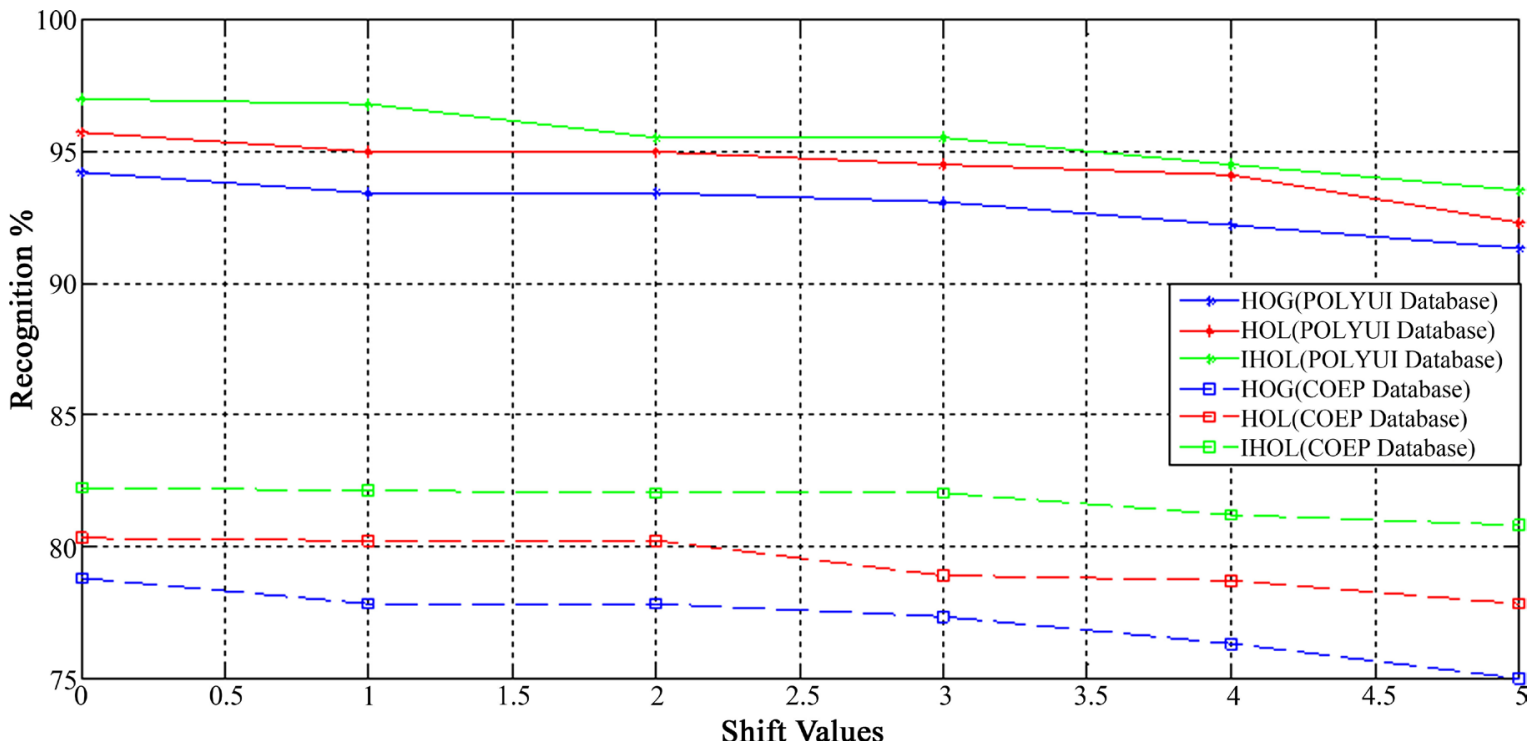

Figure 10. Recognition rate for different shift values on POLYU I/COEP database. 


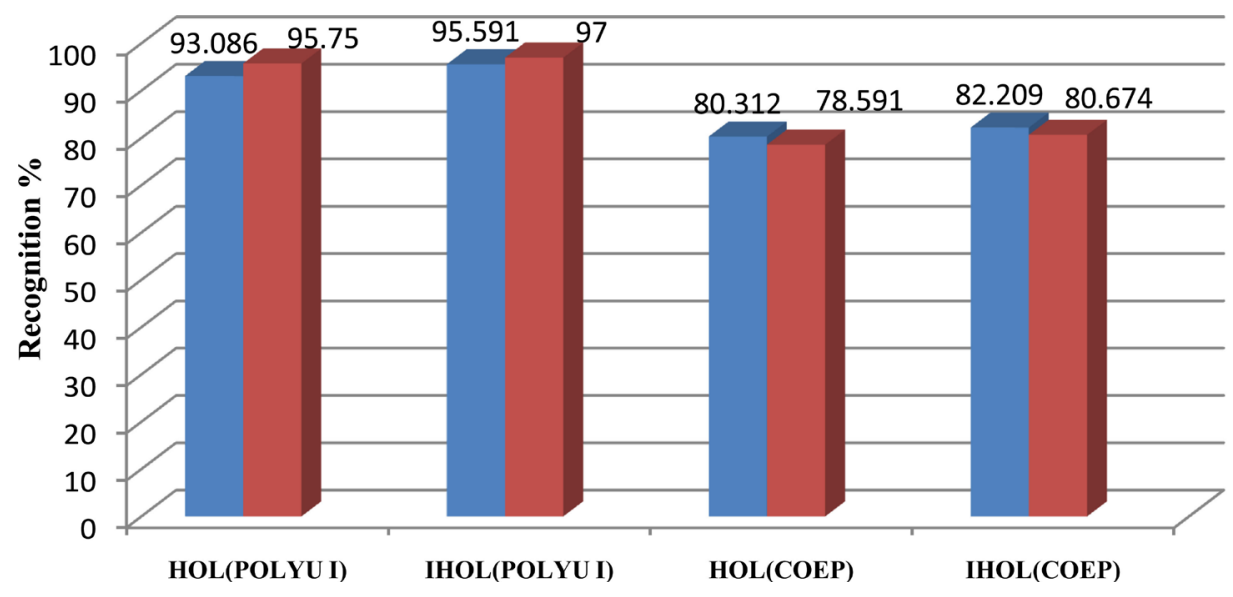

Euclidean

Consine

Figure 11. Recognition rate for POLYU I/COEP database at different distance measure.

algorithm has attain good recognition rate when compare to $\mathrm{HOL}$ algorithm in COEP database. In the palmprint classification stage, matching features using the Euclidean distance is far better than Cosine distance on COEP database.

\section{Conclusion}

This paper investigated how to further increase the recognition performance of palm print recognition system. To achieve this, we have brought certain modifications to the HOL algorithm which increases the recognition rate. This improved HOL algorithm is robust to illumination changes, small variations in translation and rotation. It has the better discrimination ability than the previous HOL algorithm for palm print identification. Thus, our IHOL together with the subspace learning method such as PCA can enhance the recognition performances on PolyU I database and COEP database. In our future work, we intend to use same strategies for palm print verification. Similarly, we use certain strategies to increase the recognition rate of COEP database and by adopting various subspace learning methods.

\section{References}

[1] Jain, A., Bolle, R. and Pankanti, S. (1999) Biometrics: Personal Identification in Networked Society Boston. Kluwer Academic Publishers, Mass.

[2] Zhang, D., Kong, W.K., You, J. and Wong, M. (2003) On-Line Palmprint Identification. IEEE Transaction PAMI, 25, 1041-1050.

[3] Zhang, D. and Shu, W. (1999) Two Novel Characteristics in Palmprint Verification: Datum Point Invariance and Line Feature Matching. Pattern Recognition, 32, 691-702. http://dx.doi.org/10.1016/S0031-3203(98)00117-4

[4] Duta, N., Jain, A.K. and Mardia, K.V. (2001) Matching of Palmprint. Pattern Recognition Letters, 23, 477-485.

[5] Li, W., Zhang, D. and Xu, Z. (2002) Palmprint Identification by Fourier Transform. International Journal of Pattern Recognition and Artificial Intelligence, 16, 417-432. http://dx.doi.org/10.1142/S0218001402001757

[6] Lu, G., Zhang, D. and Wang, K. (2003) Palmprint Recognition Using Eigenpalms Features. Pattern Recognition Letters, 24, 1463-1467. http://dx.doi.org/10.1016/S0167-8655(02)00386-0

[7] You, J., Kong, W.K., Zhang, D. and Cheung, K. (2004) On Hierarchical Palmprint Coding with Multi-features for Personal Identification in Large Databases. IEEE Transactions on Circuit Systems for Video Technology, 14, 234-243. http://dx.doi.org/10.1109/TCSVT.2003.821978

[8] Zhang, L. and Zhang, D. (2004) Characterization of Palmprints by Wavelet Signatures via Directional Context Modeling. IEEE Transactions on SMC-B, 34, 1335-1347. http://dx.doi.org/10.1109/TSMCB.2004.824521

[9] Kong, W.K. and Zhang, D. (2004) Feature-Level Fusion for Effective Palmprint Authentication. Proceeding of the 1st ICBA, LNCS 3072, Hong Kong, 15-17 July 2004, 761-767.

[10] Kong, A. and Zhang, D. (2004) Competitive Coding Scheme for Palmprint Verification. Proceedings of the 17th International Conference on Pattern Recognition (ICPR), Cambridge, UK, 23-26 August 2004, 520-523.

[11] Asha, S. and Chellappan, C. (2013) Statistical Line Based Palmprint Recognition Using Elliptical Gabor Filters. Jour- 
nal of Theoretical and Applied Information Technology, 58, 121-129.

[12] Sun, Z., Tan, T., Wang Y. and Li, S.Z. (2005) Ordinal Palmprint Representation for Personal Identification. 2005 IEEE Computer Society Conference on Computer Vision and Pattern Recognition (CVPR' 05), 1, 279-284. http://dx.doi.org/10.1109/CVPR.2005.267

[13] Belhumeur, P., Hespanha, J. and Kriegman, D. (1997) Eigenfaces vs. Fisherfaces: Recognition Using Class Specific Linear Projection. IEEE Transaction Pattern Analysis Machine Intellegence, 19, 711-720.

[14] Prasad, S.M., Govindan, V.K. and Sathidevi, P.S. (2009) Palmprint Authentication Using Fusion of Wavelet Based Representation. IEEE Coimbatore, Coimbatore, 9-11 December 2009, 520-525.

[15] Ojala, T., Pietikainen, M. and Harwood, D. (1996) A Comparative Study of Texture Measures with Classification Based on Feature Distributions. Pattern Recognition, 29, 51-59. http://dx.doi.org/10.1016/0031-3203(95)00067-4

[16] Ojala, T., Pietikainen, M. and Maenpaa, T. (2002) Multi Resolution Gray-Scale and Rotation Invariant Texture Classification with Local Binary Patterns. IEEE Transactions on Pattern Analysis and Machine Intelligence, 24, 971-987. http://dx.doi.org/10.1109/TPAMI.2002.1017623

[17] Meng, J., Gao, Y. and Wang, X. (2010) Face Recognition Based on Local Binary Patterns with Threshold. IEEE International Conference on Granular Computing, San Jose, 14-16 August 2010, 352-356. http://dx.doi.org/10.1109/grc.2010.72

[18] Tan, X. and Triggs, B. (2010) Enhanced Local Texture Feature Sets for Face Recognition under Difficult Lighting Conditions. IEEE Transactions on Image Processing, 19, 1635-1650. http://dx.doi.org/10.1109/TIP.2010.2042645

[19] Zhang, B., Gao, Y., Zhao, S. and Liu, J. (2010) Local Derivative Pattern versus Local Binary Pattern: Face Recognition with High-Order Local Pattern Descriptor. IEEE Transaction on Image Processing, 19, 533-544. http://dx.doi.org/10.1109/TIP.2009.2035882

[20] Zhang, W.G., Shan, S.G., Gao, W. and Zhang, H.M. (2005) Local Gabor Binary Pattern Histogram Sequence (LGBPHS): A Novel Non-Statistical Model for Face Representation and Recognition. Proceedings of IEEE International Conference on Vision, 17-21 October 2005, 786-791.

[21] Li, M. and Staunton, R.C. (2008) Optimum Gabor Filter Design and Local Binary Patterns for Texture Segmentation. Pattern Recognition Letters, 29, 664-672. http://dx.doi.org/10.1016/j.patrec.2007.12.001

[22] Ekinci, M. and Aykut, M. (2007) Gabor-Based kernel PCA for Palmprint Recognition. Electron Letter, 43, $1077-1079$. http://dx.doi.org/10.1049/el:20071688

[23] Chu, R., Lei, Z., Han, Y., He, R. and Li, S.Z. (2007) Learning Gabor Magnitude Features for Palmprint Recognition. Processing of 8th Asian Conference on Computer Vision - Part II, Tokyo, 18-22 November 2007, 22-31. http://dx.doi.org/10.1007/978-3-540-76390-1 3

[24] Jia, W., Huang, D.S., Tao, D. and Zhang, D. (2008) Palmprint Identification Based on Directional Representation. Proceeding. IEEE International Conference SMC, Singapore, 12-15 October 2008, 1562-1567.

[25] Dalal, N. and Triggs, B. (2005) Histograms of Oriented Gradients for Human Detection. Proceeding of 2005 IEEE Computer Society Conference on Computer Vision and Pattern Recognition, San Diego, 25-25 June 2005, 886-893. http://dx.doi.org/10.1109/cvpr.2005.177

[26] Jia, W., Xiang, R., Lei, Y., Yang, Z. and Gui, J. (2014) Histogram of Oriented Lines for Palmprint Recognition. IEEE Transactions on Systems, Man, and Cybernetics: Systems, 44, 385-395. http://dx.doi.org/10.1109/TSMC.2013.2258010 